\title{
On the Dependence of UWB Impulse Radio Link Performance on Channel Statistics
}

\author{
R. D. Wilson and R. A. Scholtz \\ Communication Sciences Institute \\ University of Southern California \\ Los Angeles CA 90089-2565 \\ robert.wilson@usc.edu,scholtz@usc.edu
}

\begin{abstract}
An important set of applications for ultra-wideband radio involves high data rate communication in indoor, and thus highly cluttered, multipath environments. Hence the statistical model of the multipath channel is an important factor in the evaluation of radio designs. The model should be realistic enough to provide accurate performance estimates, while also simple enough to facilitate rapid simulation and tractable analysis. In this paper we present two simplifications to the standard multipath model and show that both closely reproduce the performance of the standard model for a variety of radio configurations, thus providing lower complexity alternatives for radio simulation or analysis. The characteristics of each model are then compared to gain some insight into the impact of different channel metrics on radio performance.
\end{abstract}

\section{INTRODUCTION}

Ultra-wideband impulse radio (UWB-IR) uses very short carrier-less pulses with bandwidths on the order of a few Gigahertz to form a communications link [1]. The United States Federal Communications Commission has issued a First Report and Order [2] regulating the use of UWB devices, effectively restricting them to the band between 3.1 and 10.6 $\mathrm{GHz}$. Due to the very wide bandwidths involved the channel models developed for traditional radios were considered inadequate, or at least unreliable, for evaluating ultra-wideband links, and hence a number of researchers embarked upon new measurement campaigns over the frequency range relevant to UWB radios [3][4]. As a result of these measurements statistical models have been suggested for the UWB channel [5][6] that do not differ greatly from those commonly used for narrowband evaluations and the model put forth by Saleh and Valenzuela [7] in particular.

One of the common differences between UWB models and the Saleh and Valenzuela model is the use of a log-normal rather than Rayleigh distribution to describe the statistics of the multipath amplitudes, the log-normal generally having been found to more accurately match the observed data. However, a careful reading of the original work [7] will show that the authors of the famous narrowband model also found a lognormal distribution to be a more accurate match to observed data, but chose to use the Rayleigh due to its greater simplicity,

This work was supported in part by the Integrated Media Systems Center, an NSF Research Engineering Research Center, and by the MURI Project under Contract DAAD19-01-1-0477. its concordance with the understanding of the physical process of fading, and the suspected inadequacies of the measurement process. This is not to say that the use of a log-normal distribution is wrong, in fact it is the existence of compelling arguments for both distributions that has inspired us to explore in this paper how strongly the performance of a UWB-IR system depends on the particular channel model used.

In the sections below we detail the possible models, the parameters on which they depend and the characteristics of the resulting random processes. Apart from the choice between Rayleigh and log-normal amplitude distributions we also consider the modelling of arrival times as a single- or dual-mode poisson process, the latter model often being used to capture the observed clustering behaviour of multipath. Although this behaviour is well documented by many experimenters, the question we aim to answer here is whether omitting it from our model will critically alter the simulated performance of a radio. Clearly a radio's performance is a function of the channel it faces in some respect, so having asked these questions we are faced with another: if the particular distribution function of the channel is not critical, then what properties are fundamental to determining performance? Common metrics such as maximum delay and delay spread will be considered, as well as mean multipath arrival rate and energy percentiles in terms of numbers of paths.

\section{Ultra-Wideband Signal And Receiver Models}

The general transmitted UWB waveform representing the user's $n^{\text {th }}$ data symbol is described by

$$
s\left(d_{n}(u), t\right)=\sqrt{\frac{E_{\mathrm{s}}}{N_{\mathrm{pps}}}} \sum_{k=n N_{\mathrm{pps}}}^{(n+1) N_{\mathrm{pps}}-1} a_{k}^{\left(\mathrm{ds}, d_{n}\right)} p\left(t-\tau_{k}^{\left(\mathrm{th}, d_{n}\right)}\right)
$$

where $a_{k}^{\left(\mathrm{ds}, d_{n}\right)}$ is the amplitude modulation on pulse $k$ due to the $n^{\text {th }}$ data symbol $d_{n}(u)$ and the direct sequence multiple access code of the user, $\tau_{k}^{\left(\mathrm{th}, d_{n}\right)}$ is the delay on pulse $k$ due to $d_{n}(u)$ and the time hopping multiple access code of the user, $N_{\mathrm{pps}}$ is the number of pulses transmitted per data symbol, $p(t)$ is the elemental, unit energy, transmitted UWB pulse function and $E_{\mathrm{s}}$ is the energy per symbol. Dependence on $u$ indicates that a function is a random variable. The data and 
spreading sequence dependent parts of $a_{k}^{\left(\mathrm{ds}, d_{n}\right)}$ and $\tau_{k}^{\left(\mathrm{th}, d_{n}\right)}$ can be expressed explicitly as $a_{k}^{\left(\mathrm{ds}, d_{n}\right)}=a_{k}^{\mathrm{ds}} a_{k}^{d_{n}}$ and $\tau_{k}^{\left(\mathrm{th}, d_{n}\right)}=$ $\tau_{k}^{\text {th }}+\tau_{k}^{d_{n}}=c_{k}^{\text {th }} T_{\mathrm{c}}+c_{k}^{d_{n}} \delta_{\text {mod }}$, where $T_{\mathrm{c}}$ is called the chip period and $\delta_{\text {mod }}$ is a fixed time delay that can be optimized for the pulse shape $p(t)$. Let us also define the duration of $s\left(d_{n}(u), t\right)$ as $T_{s}$ and an integer $N_{\mathrm{cps}}=T_{\mathrm{s}} / T_{\mathrm{c}}$ as the number of chips per symbol. We will restrict ourselves to binary modulation, i.e., $d_{n} \in\{0,1\}$, and to short code systems, where the period of the spreading sequence, that is of $a_{k}^{\mathrm{ds}}$ or $c_{k}^{\text {th }}$, is equal to number of chips per symbol $\left(N_{\mathrm{ssp}}=N_{\mathrm{cps}}\right)$.

The signal observed at the receiver is the convolution of the signal of equation (1) with some discrete multipath channel, the models for which will be discussed in more detail later. The received signal representing the $n^{\text {th }}$ data symbol is given by

$$
r\left(u, d_{n}(u), t\right)=\sum_{l=0}^{L-1} g_{l}(u) s\left(d_{n}(u), t-\tau_{l}(u)\right)
$$

where $g_{l}(u)$ and $\tau_{l}(u)$ are the random channel gains and delays respectively, and any distortive effects due to antennas and other elements are implicity included in the pulse shape $p(t)$.

We will assume that for a sufficient number of users multiple access interference can adequately be approximated as Gaussian by appeal to the central limit theorem and hence if desired can be accounted for by an increase in noise variance. Thus the signal observed at the receiver is the sum of (2) and white Gaussian noise, specifically

$$
r(u, t)=\sum_{n} r\left(u, d_{n}(u), t\right)+w(u, t)
$$

where $w(u, t)$ is Gaussian with flat two-sided power spectral density $N_{o} / 2$.

Because we will consider only binary symbols the matched filter receiver needs a single correlator with template equal to the difference of the transmitted symbol waveforms. To take advantage of multipath diversity the Rake receiver structure will be considered and the maximal ratio combining (MRC) rule used to form the decision variable passed to a threshold function. Consider a Rake receiver with $N_{\mathrm{r}}$ correlators, the $j^{\text {th }}$ correlator has template function given by

$$
\begin{aligned}
h_{j}(t)= & \frac{1}{\sqrt{N_{\mathrm{pps}}}} \sum_{k=n N_{\mathrm{pps}}}^{(n+1) N_{\mathrm{pps}}-1} a_{k}^{\mathrm{ds}} \\
& \cdot\left(a_{k}^{0} p\left(t-\tau_{k}^{0}-\tau_{f(j)}\right)-a_{k}^{1} p\left(t-\tau_{k}^{1}-\tau_{f(j)}\right)\right)
\end{aligned}
$$

where $f(j)$ is the multipath component to which the $j^{\text {th }}$ correlator is synchronized, here we will use the $N_{r}$ strongest components, and the receiver makes the decision

$$
\hat{d}_{n}=\operatorname{sgn}\left(Z_{n}(u)+N(u)\right)
$$

where $\operatorname{sgn}(x)$ is the sign of $x, N(u)$ is gaussian with variance

$$
\sigma^{2}=\frac{N_{o}}{2} \int_{-\infty}^{\infty}\left(\sum_{j=0}^{N_{\mathrm{r}}-1} g_{f(j)}(u) h_{j}(t)\right)^{2} d t
$$

and

$$
\begin{aligned}
Z_{n}(u)= & \frac{\sqrt{E_{\mathrm{s}}}}{N_{\mathrm{pps}}} \sum_{j=0}^{N_{\mathrm{r}}-1} g_{f(j)}(u) \int_{-\infty}^{\infty} h_{j}(t) r(t) d t \\
= & \sum_{j=0}^{N_{\mathrm{r}}-1} \sum_{l=0}^{L-1} g_{f(j)}(u) g_{l}(u) \\
& \cdot \sum_{m} \sum_{k=m N_{\mathrm{pps}}}^{(m+1) N_{k_{\mathrm{ps}}}-1(n+1) N_{\mathrm{pps}}-1} a_{k}^{\mathrm{ds}} a_{k^{\prime}}^{\mathrm{ds}} a_{k}^{d_{m}} \\
& \cdot\left(a_{k^{\prime}}^{0} R_{\mathrm{p}}\left(\tau_{f(j)}(u)-\tau_{l}(u)+\tau_{k^{\prime}}^{(\mathrm{th}, 0)}-\tau_{k}^{\left(\mathrm{th}, d_{m}\right)}\right)\right. \\
& \left.-a_{k^{\prime}}^{1} R_{\mathrm{p}}\left(\tau_{f(j)}(u)-\tau_{l}(u)+\tau_{k^{\prime}}^{(\mathrm{th}, 1)}-\tau_{k}^{\left(\mathrm{th}, d_{m}\right)}\right)\right)
\end{aligned}
$$

where $R_{\mathrm{p}}(\tau)=\int_{-\infty}^{\infty} p(t-\tau) p(t)$. We will assume that the random gains $g_{f(j)}(u)$ which are required by the receiver to perform maximal ratio combining are perfectly estimated.

\section{Performance Analysis}

Given the channel amplitudes and delays and the data, $Z_{n}(u)$ is deterministic. Writing $Z_{n}(\mathbf{g}, \boldsymbol{\tau}, \mathbf{d})$ to indicate $Z_{n}(u)$ when the channel and data are known, the conditional probability of bit error is given by

$$
P_{\mathrm{e} \mid \mathbf{g}, \boldsymbol{\tau}, \mathbf{d}}=P\left(\hat{d}_{n} \neq d_{n} \mid \mathbf{g}, \boldsymbol{\tau}, \mathbf{d}\right)=Q\left(\frac{Z_{n}(\mathbf{g}, \boldsymbol{\tau}, \mathbf{d})}{\sigma}\right)
$$

and the probability of error averaged over all channels is given by

$$
P_{\mathrm{e} \mid \mathbf{d}}=\mathbb{E}_{\mathbf{g}, \boldsymbol{\tau}}\left\{P_{\mathrm{e} \mid \mathbf{g}, \boldsymbol{\tau}, \mathbf{d}}\right\} .
$$

We are often interested in knowing more about a radio's performance than just its probability of error averaged over all possible channels, for example, the probability of the radio experiencing a channel such that its bit error rate drops below a certain threshold, known as the outage probability. For threshold $\theta$ the outage probability is given by

$$
P_{\text {outage } \mid \mathbf{d}}=\iint_{P_{e \mid \mathbf{g}, \boldsymbol{\tau}, \mathbf{d}}>\theta} p_{\mathbf{g}, \boldsymbol{\tau}}(\mathbf{g}, \boldsymbol{\tau}) d \mathbf{g} d \boldsymbol{\tau}
$$

where $p_{\mathbf{g}, \boldsymbol{\tau}}(\mathbf{g}, \boldsymbol{\tau})$ is the joint probability density function of all channel amplitudes and delays.

In this study we will consider three different multiple access and modulation configurations. Two use time hopping codes and encode data using bit-flipping and pulse position modulation respectively, the third uses direct sequence coding 
and bit-flipping modulation. For the time hopping sequence we will assume the set of $c_{k}^{\text {th }}$ 's are chosen to be the elements of an $\left\{N_{\mathrm{cps}}, N_{\mathrm{pps}}, 1\right\}$ difference set, the key property of such a set being that if any integer not equivalent to zero is added modulo $N_{\text {cps }}$ to all $N_{\text {pps }}$ elements of the set, the new set has exactly 1 element in common with the original set, so that any time shift of the time hopped sequence will intersect with the original sequence in exactly one chip location. The direct sequence code is taken to be a Gold code, having a three valued correlation function.

Let us assume that the chip duration $T_{\mathrm{c}}$ is greater than or equal to twice the support of $p(t)$, with the result that all transmitted pulses are at least one pulse width apart. Express the multipath inter-arrival time as $\tau_{f(j)}-\tau_{l}=\alpha_{j, l} T_{\mathrm{c}}+\beta_{j, l}$ where $\alpha_{j, l}=\left[\frac{\tau_{f(j)}-\tau_{l}}{T_{\mathrm{c}}}\right]$ and $-0.5 T_{\mathrm{c}} \leq \beta_{j, l}<0.5 T_{\mathrm{c}}$. For pulse position modulation let $c_{k}^{d_{n}}=\left(k+d_{n}\right)(\bmod 2)$ and for bit flipping $a_{k}^{d_{n}}=(-1)^{d_{n}}$, then with time hopped coding we have that

$$
\begin{aligned}
Z_{n}^{\mathrm{th}}(u)=( & -1)^{d_{n}} \sqrt{E_{\mathrm{s}}} \sum_{j=0}^{N_{\mathrm{r}}-1} \sum_{\substack{l=0 \\
\alpha_{j, l}=0}}^{L-1} g_{f(j)}(u) g_{l}(u) X_{\bmod }\left(\beta_{j, l}\right) \\
& +\frac{\sqrt{E_{\mathrm{s}}}}{N_{\mathrm{pps}}} \sum_{j=0}^{N_{\mathrm{r}}-1} \sum_{\substack{l=0 \\
\alpha_{j, l} \neq 0}}^{L-1} g_{f(j)}(u) g_{l}(u) X_{\bmod }\left(\beta_{j, l}\right)
\end{aligned}
$$

where for PPM $X_{\text {mod }}=X_{\mathrm{ppm}}$ is given by

$$
X_{\mathrm{ppm}}\left(\beta_{j, l}\right)=R_{\mathrm{p}}\left(\beta_{j, l}\right)-\frac{R_{\mathrm{p}}\left(\beta_{j, l}+\delta_{\mathrm{mod}}\right)+R_{\mathrm{p}}\left(\beta_{j, l}-\delta_{\mathrm{mod}}\right)}{2}
$$

and for bit flipping $X_{\mathrm{mod}}=X_{\mathrm{bf}}$ is given by

$$
X_{\mathrm{bf}}\left(\beta_{j, l}\right)=2 R_{\mathrm{p}}\left(\beta_{j, l}\right) .
$$

The reader is referred to [8] for further details. In (11),(12) and (13) we have implicitly assumed that $d_{m}$ is the same for all $m$, for bit flipping this is the worst case scenario in that the variance of $Z_{n}(u)$ is maximized; for PPM the effect of changing data is to make the number of $R_{p}\left(\beta_{j, l}+\delta_{m o d}\right)$ and $R_{p}\left(\beta_{j, l}-\delta_{\text {mod }}\right)$ terms not quite equal, which should not significantly affect the outcome.

For direct sequence with bit flipping modulation we have

$$
\begin{aligned}
Z_{n}^{\mathrm{ds}}(u) & =(-1)^{d_{n}} \sqrt{E_{\mathrm{s}}} \sum_{j=0}^{N_{\mathrm{r}}-1} \sum_{\substack{l=0 \\
\alpha_{j, l}=0}}^{L-1} g_{f(j)}(u) g_{l}(u) X_{\mathrm{bf}}\left(\beta_{j, l}\right) \\
& +\frac{\sqrt{E_{\mathrm{s}}}}{N_{\mathrm{pps}}} \sum_{j=0}^{N_{\mathrm{r}}-1} \sum_{\substack{l=0 \\
\alpha_{j, l} \neq 0}}^{L-1} g_{f(j)}(u) g_{l}(u) X_{\mathrm{bf}}\left(\beta_{j, l}\right) C_{\mathrm{ds}}\left(\alpha_{j, l}\right)
\end{aligned}
$$

where

$$
C_{\mathrm{ds}}\left(\alpha_{j, l}\right)=\sum_{k^{\prime}=0}^{N_{\mathrm{pps}}-1 \ominus \alpha_{j, l}} a_{k^{\prime}}^{\mathrm{ds}} a_{k^{\prime} \oplus \alpha_{j, l}}^{\mathrm{ds}}-\sum_{k^{\prime}=N_{\mathrm{pps}} \ominus \alpha_{j, l}}^{N_{\mathrm{pps}}-1} a_{k^{\prime}}^{\mathrm{ds}} a_{k^{\prime} \oplus \alpha_{j, l}}^{\mathrm{ds}}
$$

and all additions and subtractions of $\alpha_{j, l}$ are performed modulo $N_{\text {pps }}$. The definition of $C_{\mathrm{ds}}$ implies that the two symbol waveforms that potentially interfere with detection for a given multipath delay (one of which might be the waveform of interest interfering with its own detection) are modulated by different data, which results in the worst case interference for direct sequence coding.

\section{Ultra-Wideband Channel Models}

The reference channel will be the channel model that has been proposed for the IEEE 802.15.3a standard, currently under development [6]. Two variations on this channel will be considered, the first using Rayleigh instead of log-normal fading and the second additionally replacing the dual mode arrival process with a single mode process.

The proposed IEEE 802.15.3a model has impulse response given by

$$
c_{1}(t)=X(u) \sum_{n=0}^{N-1} \sum_{k=0}^{K-1} g_{n, k}(u) \delta\left(t-\tau_{n, k}(u)\right)
$$

where

$$
\begin{gathered}
g_{n, k}(u)=p_{n, k}(u) 10^{G_{n, k}(u) / 20}, \\
f_{G_{n, k}}(z)=\frac{1}{\sqrt{2 \pi\left(\sigma_{1}^{2}+\sigma_{2}^{2}\right)}} e^{\frac{-\left(z-\mu_{n, k}\right)^{2}}{2\left(\sigma_{1}^{2}+\sigma_{2}^{2}\right)}} \\
\mu_{n, k}=\frac{10 \ln \left(\Omega_{0}\right)-10 T_{n} / \Gamma-10 \zeta_{n, k} / \gamma}{\ln (10)}-\frac{\left(\sigma_{1}^{2}+\sigma_{2}^{2}\right) \ln (10)}{20}, \\
f\left(T_{n} \mid T_{n-1}\right)=\Lambda e^{-\Lambda\left(T_{n}-T_{n-1}\right)} \\
f\left(\zeta_{n, k} \mid \zeta_{n, k-1}\right)=\lambda e^{-\lambda\left(\zeta_{n, k}-\zeta_{n, k-1}\right)}
\end{gathered}
$$

and $\tau_{n, k}=T_{n}+\zeta_{n, k}$ is the time of arrival, $g_{n, k}=p_{n, k} \xi_{n} \beta_{n, k}$ is the amplitude coefficient of the $k^{\text {th }}$ ray in the $n^{\text {th }}$ cluster and the expression for $\mu_{n, k}$ is calculated so that $\mathbb{E}\left[\left|g_{n, k}(u)\right|^{2}\right]=\Omega_{0} e^{-T_{n} / \Gamma} e^{-\zeta_{n, k} / \gamma}$. The polarity of each arrival is determined by $p_{n, k}$ and is \pm 1 with equal probability. When generating sample channels from this distribution the total energy of the impulse response is normalized before multiplication by the shadowing term $X(u)$ where $20 \log _{10}(X(u))$ is a mean zero gaussian random variable with variance $\sigma_{x}^{2}$. This procedure and the distribution of $X(u)$ will remain the same for all channel models considered.

In the second channel model, which we will denote as $c_{2}(t)$, the log-normal amplitude distribution of $g_{n, k}(u)$ is replaced with a Rayleigh distribution

$$
f_{\left|g_{n, k}\right|}(z)=\frac{z}{\rho^{2}} e^{-\frac{z^{2}}{2 \rho^{2}}} U(z)
$$

where $U(z)$ is the unit step function and 
TABLE I

PARAMETERS FOR EQUIVALENT CHANNELS

\begin{tabular}{|l|c|c|c|}
\hline \multirow{2}{*}{ Channel parameter } & \multicolumn{2}{|c|}{ CM 1 } & CM 3 \\
\cline { 2 - 4 } & $\mathbf{c}_{\mathbf{1}}(\mathbf{t})$ & $\mathbf{c}_{\boldsymbol{3}}(\mathbf{t})$ & $\mathbf{c}_{\mathbf{1}}(\mathbf{t}), \mathbf{c}_{\mathbf{2}}(\mathbf{t})$ \\
\hline$\Lambda\left(n s^{-1}\right)$ & 0.0233 & - & 0.0667 \\
\hline$\lambda\left(n s^{-1}\right)$ & 2.5 & 3.8 & 2.1 \\
\hline$\Gamma(n s)$ & 7.1 & - & 14 \\
\hline$\gamma(n s)$ & 4.3 & 3.9 & 7.9 \\
\hline$\sigma_{1}(\mathrm{~dB})$ & 3.3941 & 4.8 & 3.3941 \\
\hline$\sigma_{2}(\mathrm{~dB})$ & 3.3941 & - & 3.3941 \\
\hline$\sigma_{x}(\mathrm{~dB})$ & 3 & 3 & 3 \\
\hline
\end{tabular}

$$
\rho=\sqrt{\frac{\Omega_{0}}{2}} e^{-\frac{T_{n}}{2 \Gamma}} e^{-\frac{\zeta_{n, k}}{2 \gamma}} .
$$

Choosing $\rho$ according to (18) ensures that $\mathbb{E}\left[\left|g_{n, k}(u)\right|^{2}\right]$ is the same as in the log-normal case and can also be shown to minimize the Kullback-Leibler distance between the two distributions.Note that the distribution of $\left|g_{n, k}(u)\right|$ is a function of a single parameter and $\sigma_{1}$ and $\sigma_{2}$ have been eliminated.

The third model, denoted $c_{3}(t)$, uses Rayleigh fading and in addition neglects the clustering of paths. The channel model can be defined using (16), (17) and (18) with $N=1$ and $T_{n}=\sigma_{2}=0$.

\section{RESULts}

Bit error rate and outage probability performance were evaluated as shown in section III, with integrals over channels being done by Monte-Carlo simulation using the models of section IV. Two sets of parameters were chosen for model $c_{1}(t)$, corresponding to channel types CM 1 and CM 3 in [6]. The pulse shape was a sinusoid of approximately $7.4 \mathrm{GHZ}$ with a Gaussian envelope optimized to maximize energy while ensuring compliance with the FCC mask, the resulting pulse has duration of approximately $0.5 \mathrm{~ns}$. The optimal value of $\delta_{m o d}$, which maximizes $X_{p p m}(0)$, is $0.06 n s$, possibly an unrealistic requirement for a practical system but we will assume it is achieved for the theoretical radio of this experiment, resulting in $X_{p p m}(0)=1.8$. For time hopping $N_{p p s}=8$ and in all cases $N_{\text {cps }}=63$.

Given that one motivation for considering alternative channel models is reduce simulation complexity, it is germane to point out that in this experiment simulations using channel model $c_{3}(t)$ were completed significantly faster than simulations using the other models. The precise advantage is dependent on the computer code and machine and we will not attempt to quantify it here.

Using the parameters given in Table I for each channel model good visual agreement between bit error rate and outage probability curves was found between all three channel types for each data rate and each modulation and spreading format.

In Fig. 1 bit error rate curves when $T_{c}=1.25 \mathrm{~ns}$, corresponding to a data rate of $12.7 \mathrm{Mbps}$, are shown for direct

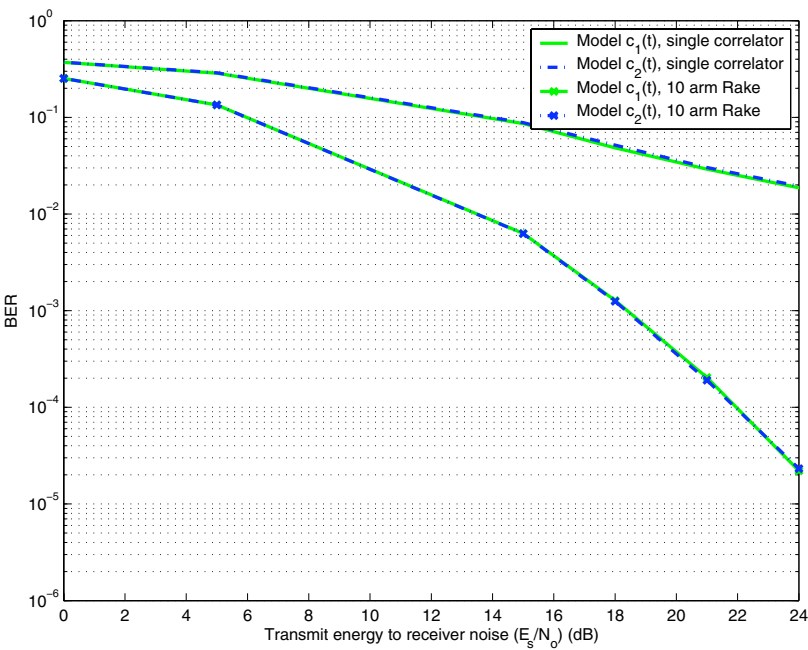

Fig. 1. Bit error rates using direct sequence and bit flipping modulation for channel type CM 3.

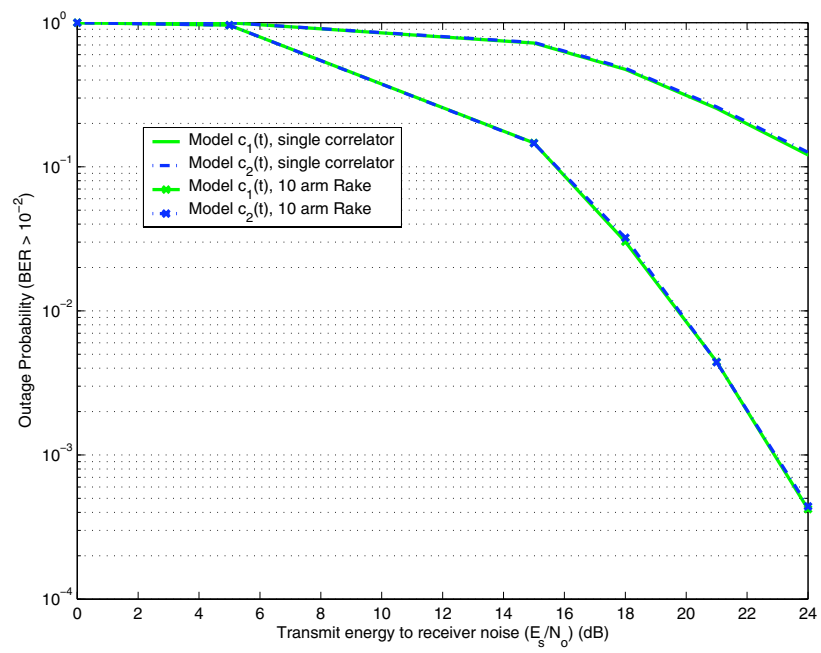

Fig. 2. Outage probability using direct sequence and bit flipping modulation for channel type CM 3.

sequence spreading with bit-flipping and a Rake receiver using 1 and 10 arms under channel models $c_{1}(t)$ and $c_{2}(t)$, where the channel is of type CM 3. Fig. 2 shows the corresponding outage probabilities.

In Fig. 3 bit error rate curves are compared over a channel of type CM 1 using models $c_{1}(t)$ and $c_{3}(t)$ for time hopped spreading with PPM and a Rake receiver using 1 and 10 arms, when the data rate is $3.17 \mathrm{Mbps}$. Fig. 4 shows the corresponding outage probabilities.

Given that these channels each demonstrate similar effects on a variety of UWB radio links, it would be nice to know what the channels have in common. In Table II the mean excess delay, maximum excess delay, RMS delay spread, mean path inter-arrival time (IAT), number of paths within $10 \mathrm{~dB}$ of the peak $\left(N P_{10 d B}\right)$ and number of paths composing $85 \%$ of the 


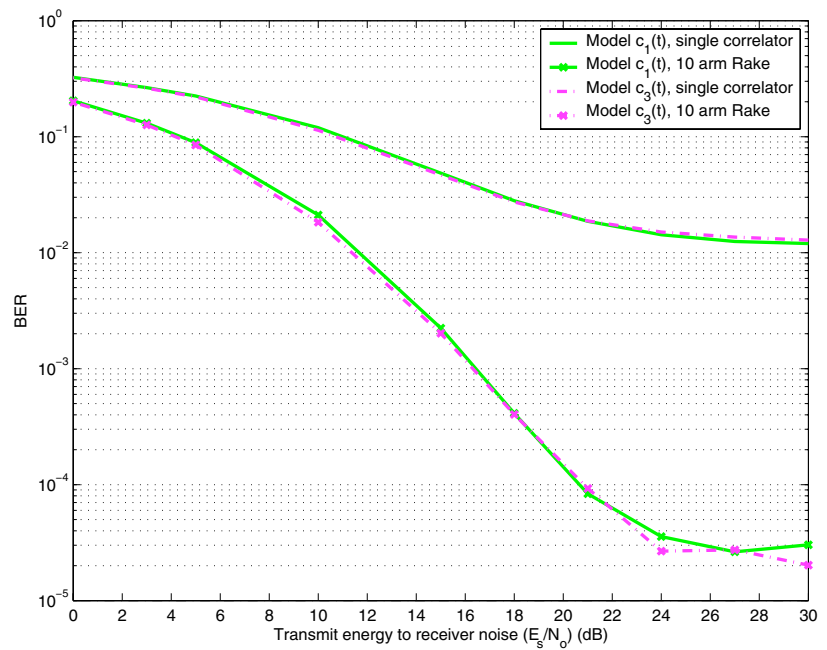

Fig. 3. Bit error rates using time hopping and PPM under channel model CM 1.

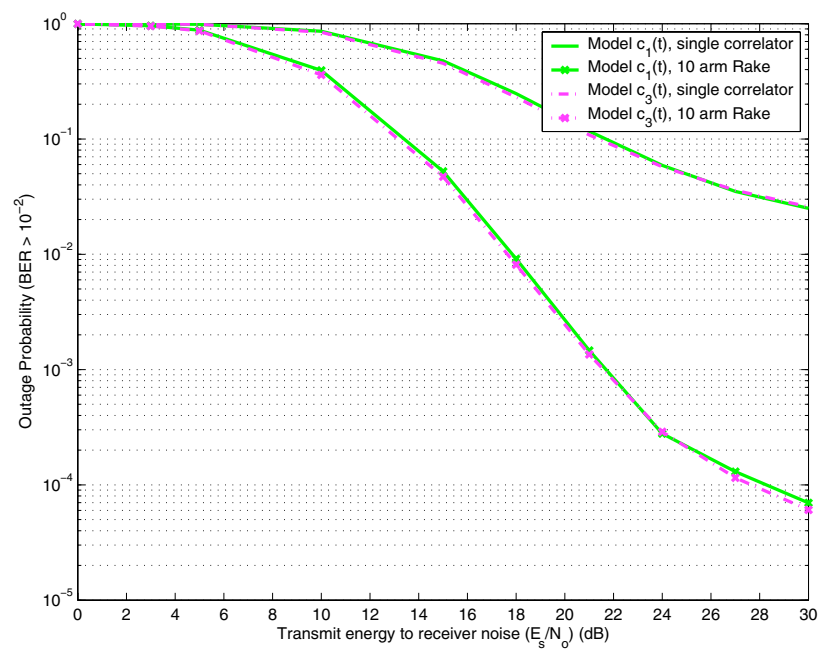

Fig. 4. Outage probabilities using time hopping and PPM under channel model CM 1.

channel energy $\left(N P_{85 \%}\right)$ are shown for each channel model for channel type CM 1. Each metric is an average over 100 sample channels.

Channel models $c_{1}(t)$ and $c_{2}(t)$ are close in all measures, thus it would seem substituting a Rayleigh amplitude distribution for a log-normal does not significantly alter the underlying characteristics of the channel. Model $c_{3}(t)$ without clustered arrivals has notably lower delay spread measures than the other models, but the mean arrival rate and energy percentile measures are somewhat similar to those of the models with clustered arrivals. Although it is not possible to draw any universal conclusions from this single experiment, it is clear that mean delay spread measures alone are not sufficient to predict performance over a multipath channel, and in fact other
TABLE II

CHANNEL CHARACTERISTICS

\begin{tabular}{|l|c|c|c|}
\hline Channel metric & $\mathbf{c}_{\boldsymbol{1}}(\mathbf{t})$ & $\mathbf{c}_{\boldsymbol{2}}(\mathbf{t})$ & $\mathbf{c}_{\boldsymbol{3}}(\mathbf{t})$ \\
\hline mean excess delay (ns) & 5.0 & 5.1 & 3.8 \\
\hline max. excess delay (ns) & 76.0 & 81.1 & 38.7 \\
\hline RMS delay spread (ns) & 5.4 & 5.6 & 3.9 \\
\hline mean path IAT (ns) & 0.32 & 0.3 & 0.27 \\
\hline $\mathrm{NP}_{10 d B}$ & 17 & 16.7 & 18 \\
\hline $\mathrm{NP}_{85 \%}$ & 22 & 23.4 & 21 \\
\hline
\end{tabular}

characteristics such as mean arrival rate may be more decisive.

\section{CONCLUSIONS}

The bit error rate and outage probability of three ultrawideband radios, using bit flipped modulation with direct sequence and time-hopped spreading and pulse position modulation with time-hopped spreading respectively, were evaluated over three alternative channel models. It was shown that, with appropriately selected parameters, multipath channel models featuring clustered arrivals with log-normal fading, clustered arrivals with Rayleigh fading and non-clustered arrivals with Rayleigh fading, all result in similar simulated performance for each UWB radio configuration. The latter two models provide potential simplifications to the evaluation of UWB radios over multipath channels for both analysis and simulation.

Some of the average characteristics of channels from each model were compared and it was found that measures such as mean excess delay and mean RMS delay spread can be quite different for channels that nonetheless result in the same radio performance. For the channels considered here, more significant criteria appeared to be mean multipath inter-arrival time and the number of paths capturing $85 \%$ of the channel energy.

\section{REFERENCES}

[1] M. Z. Win and R. A. Scholtz, "Impulse radio: How it works," IEEE Communications Letters, vol. 2, pp. 36-38, Feb. 1998.

[2] Federal Communications Commission, "Revision of part 15 of the commission's rules regarding ultra-wideband transmission systems: First report and order," April 2002. ET-Docket 98-153.

[3] R. J.-M. Cramer, R. A. Scholtz, and M. Z. Win, "Evaluation of the propagation characteristics of ultra-wideband communication channels," in International Antennas and Propagation Symposium, vol. 1, pp. 626630, IEEE, June 1998.

[4] C. Prettie, D. Cheung, L. Rusch, and M. Ho, "Spatial correlation of UWB signals in a home environment," in Proc. Ultra-Wideband Systems and Technology, pp. 65-69, IEEE, 2002.

[5] R. J.-M. Cramer, An Evaluation of Ultra-Wideband Propagation Channels. $\mathrm{PhD}$ thesis, University of Southern California, Los Angeles, California, 2000.

[6] J. R. Foerster, "Channel modeling sub-committee report (final)," Tech. Rep. P802.15-02/368r5-SG3a, IEEE P802.15 Working Group for Wireless Personal Area Networks (WPANs), December 2002. http://grouper.ieee.org/groups/802/15/pub/2002/Nov02/02490r0P80215_SG3a-Channel-Modeling-Subcommittee-Report-Final.zip.

[7] A. A. M. Saleh and R. A. Valenzuela, "A statistical model for indoor multipath propagation," J. Selected Areas of Communications, vol. 5, pp. 128-137, Feb. 1987.

[8] R. D. Wilson and R. A. Scholtz, "Comparison of CDMA and modulation schemes for UWB radio in a multipath environment," in Proc. Globecom, to be published, IEEE, 2003. 